\title{
Gerencia y Administración Financiera *
}

\section{Juan Antonio Garde Roca **}

\section{Introducción}

Asistimos a un proceso de transformación de la Administración y la gestión de lo público, como resultado de la actuación de cuatro factores básicos:

1) Una nueva configuración de la relación entre lo público y lo político a través del papel mediador que la Administración Pública cumple entre el poder político y la sociedad civil en democracia.

2) Un cambio en el alcance y dimensión de las políticas públicas, vinculado a la existencia de una pluralidad de agentes públicos actuantes (distintos niveles de soberania), al desarrollo creciente de los mercados y la iniciativa privada en un entorno de internacionalización, y a la revalorización de la iniciativa social (sociedad civil) en los objetivos comunitarios.

3) Un entorno de restricción presupuestaria y financiera para el Sector Público de las economías avanzadas, junto con una debilidad de recursos económicos e institucionales notable en las Sociedades en vías de desarrollo.

4) Un intenso debate ideológico y político sobre el papel de lo público y las relaciones entre el Estado, el mercado y la sociedad.

El primer factor incide en la eficiencia social, la calidad, la participación, en definitiva en la creación de valor en democracia. El segundo se articula con la "Administración relacional", vinculando políticas de regulación y coordinación entre agentes. Las restricciones económicas y financieras afectan a la forma de gestionar, a la eficiencia económica y social del gasto público y su control. Finalmente, el debate ideológico y político es omnipresente y condiciona el modelo de sector público del futuro.

Todos los aspectos aquí reseñados se interrelacionan conformando una realidad dinámica a través de la cual discurre el quehacer de la gestión pública en las sociedades contemporáneas.

\section{Papel de la Administración Pública en la sociedad actual}

La Administración Pública juega un papel crucial para la legitimidad del poder político en las sociedades contemporáneas. Aparece como un elemento de continuidad y garantía institucional de democracia, asegurando tal como he senalado una fórmula clave de mediación entre el poder político y la sociedad.

La mediación de la Administración en las sociedades avanzadas está íntimamente ligada al fenómeno de la interdependencia, presente en la estructura del poder político y en las relaciones entre este poder y la Sociedad. Siguiendo a $\mathrm{C}$. VENTRISS ${ }^{1}$ :

a) La acción pública tiene lugar en entornos en los que cualquier elemento depende de cualquier otro, y el poder está compartido por múltiples actores públicos.

b) La capacidad de cualquier autoridad o actor público, de actuar unilateralmente de forma efectiva, es muy reducida.

c) Un amplio número de consecuencias indirectas, a menudo no previstas y no buscadas, incrementan la vulnerabilidad y apertura a influencias del exterior. Los gestores públicos dependen cada vez más de otros individuos y organizaciones externas.

d) Los resultados de las elecciones políticas y de las acciones administrativas sufren frecuentes retardos, costes indirectos u ocultos, más allá de las externalidades normales. Es difícil separar las consecuencias deseables y las no deseables, y pueden producirse efectos importantes y a veces críticos que no habían sido previstos.

La gestión pública en este contexto interdependiente aparece como instrumento activo de la toma de decisiones políticas, 
al incorporar valores sociales, evaluar incidencias de las decisiones y operar como marco organizado de consenso social, integrando y promoviendo la participación de la sociedad civil en las decisiones públicas.

La Administración, insertada por tanto en el marco político a través de la acción pública, no es un espacio neutral meramente instrumental, y crea valor en democracia. TERRY ${ }^{2}$ se ha referido al gestor público marcando su carácter de elemento equilibrador de la tensión entre la necesidad de servir y la de preservar. El gestor debe responder a las demandas de los políticos, los Tribunales, los grupos de interés, los funcionarios y los ciudadanos, a la vez que debe preservar la integridad institucional. En torno a esta referencia se inscribe el contenido ético de la acción pública y el establecimiento de códigos de valores de la Responsabilidad Pública. Esta concepción incide especialmente en la necesidad de una función pública profesional que, con el necesario control legislativo y social, impida la patrimonialización corporativa, protagonice la garantía de continuidad y de seguridad jurídica capaces institucionalmente de ofrecer legitimidad para las funciones creadoras de valor en democracia.

En paralelo con estas concepciones de la Administración Pública en Democracia, se ha venido desarrollando el concepto de "gestión pública estratégica" que pone el acento en los retos de gestión a que deben hacer frente los organismos públicos y sus gestores en las modernas sociedades. Siguiendo a LAX y SABENIUS, Barzelay ${ }^{3}$ se ha referido a que los gestores públicos han de desempeñar la función de entretejer una red de acuerdo firme entre autoridades, funcionarios públicos y otros agentes. El gerente público integrará en este proceso todo lo relativo a la resolución de problemas políticos, administrativos y sociales. De esta forma, aumentará la probabilidad de que las energías sociales y del sector público se dirijan hacia actividades que creen valor a los beneficiarios directos y a la sociedad en su conjunto. El proceso de evaluación de Políticas Públicas, concebido como acción pluralista, permite igualmente contar con el conjunto de los agentes implicados. Desde esta concepción la evaluación tendrá en cuenta los diversos sistemas de valores e intereses, propiciando legitimidad política, social y administrativa a las Políticas Públicas ${ }^{4}$.

En este esfuerzo de redefinición de una nueva filosofía de la Administración, se han venido desarrollando numerosos trabajos e investigaciones conducentes a reorientar la acción práctica de la Administración Pública. El Documento aLa Administración al servicio del públicon de la O.C.D.E. viene a sintetizar la preocupación dominante de dotar a la acción pública de un marco de referencia (Administración receptiva, servicio al ciudadano) que permita el impulso de políticas eficaces y de una gestión que satisfaga más cabalmente las demandas sociales. Se pone el acento en la gestión eficaz y en los modelos de organi- zación, gestión, control y procedimentales que permitan la eficiencia social. La incorporación de una versión más estratégica y adaptativa de la acción pública y de sus políticos forma parte de los enfoques prácticos más recientes para la transformación de los modelos predominantemente burocráticos del pasado.

Tratando finalmente de sintetizar las exigencias de una moderna gestión pública en democracia, podemos clasificar en tres los requerimientos de la acción pública. La conciliación entre las distintas exigencias permite definir una filosofía pública sobre la que es posible construir la acción eficaz que la sociedad demanda.

a) Factores institucionales.

Configuran el eje central de la acción más continuada de la Administración y de su importante rol como elemento de continuidad y de garantía institucional al servicio de la legitimidad del Estado y la seguridad jurídica e igualdad en la aplicación de las leyes a los ciudadanos.

b) Factores democráticos.

Aparecen vinculados al papel de mediación que la Administración cumple entre el poder político y el ciudadano. A su operatividad como marco organizado de Consenso Social, integrando y promoviendo la participación de la sociedad civil en las decisiones públicas.

La Administración Pública aparece de esta forma no sólo como una organización de "garantía institucional" o como "suministrador" de bienes y servicios públicos, se construye como elemento de conocimiento social y aprendizaje democrático. Transfiere conocimiento social al poder político, permite la aplicación de las políticas públicas, crea tejido social.

c) Factores de eficacia, calidad y eficiencia.

La consideración global de eficacia necesita ser materializada en organizaciones, procedimientos, políticas, técnicas y medios humanos capaces de actuar compaginando calidad en el servicio público y eficiencia en el uso de los recursos públicos. Estos factores requieren en las condiciones actuales de fórmulas de interacción y coordinación adecuadas entre los diversos agentes públicos, el mercado y los ciudadanos.

Dentro de esta configuración de las Administraciones Públicas, la Administración Tributaria conforma un espacio de particular tensión y renovación.

De una parte, su papel en las sociedades contemporáneas obedece a las mismas exigencias que el conjunto de la Administración Pública.

a) La Administración Tributaria es un elemento crucial para la legitimidad del poder político en un área especialmente delicada y conflictiva: la que configura el origen de los recursos financieros esenciales del sector público. 
b) Es un instrumento al servicio de la seguridad jurídica, garantía de estabilidad, objetividad y continuidad para el ciudadano-contribuyente.

c) Es una organización para la democracia, debiendo asegurar formas de mediación eficaces entre el poder político y la sociedad que impulse el proceso de colaboración y aceptación social de las cargas que configuran el Sistema Fiscal.

No obstante, el mero enunciado anterior ya descubre el espacio epropio de la Administración Tributaria. Un espacio que conlleva exigencias muy particulares que la distinguen de las demandas a las que sirven otros organismos públicos y que serán objeto de un tratamiento posterior.

\section{Los roles de la Administración Tributaria}

En el estudio de la O.C.D.E. sobre aLa Receptividad administrativa y el Contribuyente: Un resumen para decisores ${ }^{5}$ se afirma que el servicio a prestar por las Administraciones Tributarias consiste en:

- Hacer que las obligaciones y los derechos de los contribuyentes sean muy claros en cuanto a procedimientos, formularios y lenguaje utilizado.

- Introducir al máximo las nuevas tecnologías de la información para aplicar procedimientos más simples, rápidos, precisos y fiables.

- Asegurar que el sistema fiscal sea justo y equitativo sin ser demasiado complejo, de modo que tanto los contribuyentes como los funcionarios puedan aplicarlo como es debido.

Utilizando palabras del Tribunal Constitucional español, es función de la Administración evitar que alo que unos no paguen debiendo pagarlo, lo paguen otros con más espíritu cívico o con menos posibilidades de defraudar.

Esta es una tarea que la sociedad espera que desarrolle correctamente la Administración Tributaria. No obstante, es necesario profundizar más en la misión de la Administración Tributaria y en sus principales objetivos.

Nos hemos referido anteriormente a las características propias de la Administración Tributaria en relación con el conjunto de la Administración Pública, estas exigencias "peculiares» están vinculadas en primer lugar a la naturaleza específica de su finalidad: Detraer recursos de la aeconomía privada" para suministrarlos al "poder público". Y en segundo lugar a la naturaleza de los agentes a los que sirve o sobre los que actúa. a) La sociedad en su conjunto.

b) Los distintos sectores, clases, grupos de interés de particular.

c) Los ciudadanos y entidades contribuyentes individualizados.

Por lo que respecta a su finalidad específica, adetraer recursos de la economía privada...* Nunca ha sido ésta una actividad aceptada naturalmente con independencia de los valores ideológicos o sociales dominantes en cada época histórica.

De otra parte, desde siempre los distintos sectores sociales con poder económico o político han tratado de desplazar sus cargas fiscales hacia otros estratos sociales. CERVANTEs lo recuerda cuando narra cómo los comerciantes tratan de comprar hidalguías y así obtener franquicias fiscales y VicENs VIVES, en su magnífica Historia Económica de España, recoge con reiteración cómo el campo de los impuestos está ligado al poder económico y político de las distintas clases sociales a lo largo de toda nuestra Historia. SCHUMPETER en 1922 (La Crisis Fiscal del Estado) señalaba que ael espíritu de un pueblo, su nivel cultural, su estructura social, los hechos que pueden determinar su política,... todo esto y más, está escrito con claridad en su historia fiscaln. Y es que, en efecto y sin retórica alguna, uno de los mejores puntos de partida para realizar una investigación sobre una sociedad, e incluso sobre su estructura política, es estudiar su Hacienda Pública.

De forma mucho más reciente apuntaba Francisco FERNÁNDEZ ORDóÑEz al tratar los temas del fraude fiscal que "para hacer un enjuiciamiento válido del tema del fraude desde un punto de vista ético hace falta conocer la estructura política que generan las leyes fiscales, el carácter democrático del proceso legislativo y de la fiscalización del gasto público, la racionalidad y justicia del sistema tributario y de la distribución de las cargas públicas, así como en qué clases sociales se ha desarrollado el fraude y cuáles son los juicios de valor de estas clases, y de la sociedad entera, sobre el mismo".

No es por tanto exagerado referirse a la Administración Tributaria como actuante en uno de los campos de la Administración en que con mayor nitidez es posible detectar los conflictos y contradicciones sociales. La Administración Tributaria aparece así como un área muy sensible de conflicto social y por idénticas razones como campo estratégico de búsqueda de consenso y legitimidad social en democracia.

La Administración Tributaria ejerce por tanto en un campo de acción esencialmente complejo tanto en relación con el contribuyente individual como en el contexto de las relaciones sociales.

a) La acción de la Administración Tributaria se dirige en primer lugar a la Sociedad en su conjunto. 
La percepción social sobre el grado de equidad y justicia del Sistema Tributario y de su exigencia, la interiorización como valor propio del cumplimiento fiscal voluntario, el rechazo social a las conductas defraudadoras, son elementos de primera magnitud sobre los que tiene que actuar de forma prioritaria la Administración Tributaria. Más aún, si superados los enfoques meramente recaudatorios y represivos hubiera que encontrar un objetivo estratégico a la acción de la Administración Tributaria éste no podría ser otro que el de aumentar el nivel de aceptación social del Sistema Tributario y el grado de colaboración social con el mismo.

La doble cara de la Administración Tributaria, colaboradora con los cumplidores, represiva con los defraudadores, adquiere de esta forma una dimensión nueva al estar ambas prácticas al servicio de la aceptación social del Sistema Tributario.

La propia planificación de la actividad inspectora debe efectuarse teniendo en cuenta este objetivo estratégico y la evaluación de su acción llevarse a cabo preferentemente en relación con su carácter meramente instrumental al servicio de la ejemplaridad y el cumplimiento fiscal voluntario.

El grado de colaboración y aceptación social del Sistema Tributario es, por otra parte, un fenómeno claramente interdependiente, en el que muy diversos agentes económicos y sociales actúan e influyen transformando la percepción que del fenómeno tributario tiene el conjunto de la sociedad. La capacidad de la Administración Tributaria de actuar unilateralmente en este área de "conflicto potencial" es por tanto limitada.

Su estrategia debe combinar la "acción propia", a través de la comunicación externa hacia la sociedad, con la colaboración e incidencia en aquellos sectores sociales que de forma más precisa influyen en la aceptación general del modelo tributario.

b) La Administración Tributaria no puede olvidar que el conjunto social no es otra cosa que un conglomerado de instituciones, organismos, grupos, individuos, lobbies, medios de opinión. Todos ellos marcados por intereses económicos, sociales, ideológicos... que confluyen de forma dinámica e interrelacionada articulando la realidad social.

Por ello, debe tratarse de aplicar la normativa tributaria no sólo respetando los principios constitucionales y el ordenamiento jurídico vigente, y procurando el objetivo de lograr la máxima aceptación y la colaboración social con el Sistema Tributario, sino siendo también consciente de que el logro de este último objetivo está a menudo en relación directa con su capacidad para obtener la colaboración "particularn de distintos sectores y grupos sociales específicos.
La realidad de "conflicto" que subyace al impuesto hace en ocasiones muy difícil compaginar intereses particulares con generales. No obstante lo anterior, el papel específico de la Administración Tributaria estriba en su carácter de elemento equilibrador de la tensión entre servir a los diversos grupos y sectores de ciudadanos y preservar los intereses generales y la percepción que sobre la justicia y equidad del Sistema recibe el conjunto de la Sociedad.

Será imprescindible por tanto buscar acuerdos o compromisos con los diversos sectores económicos, financieros y sociales representativos en el siempre difícil marco que compagine los intereses particulares con el interés general.

Estos acuerdos son por otra parte imprescindibles cuando se pretende una gestión eficiente, contando como cuenta la Administración Tributaria con recursos materiales y humanos escasos. De forma que, determinados tipos de acuerdos pueden servir a la vez a la gestión eficiente, a la justicia tributaria y a la seguridad jurídica de los contribuyentes, tales como evaluaciones, módulos, acuerdos previos, a título meramente ilustrativo.

c) Dentro de una actividad más interna o cotidiana, la Administración Tributaria debe ser capaz de servir con eficacia a los Ciudadanos y Entidades-contribuyentes individualizados.

El "cliente" específico de la Administración Tributaria es el ciudadano-contribuyente. Un ciudadano que acude generalmente a los servicios de la Hacienda Pública para cumplir un penoso deber -pagar el impuesto- o para solicitar la devolución del impuesto ya pagado. Se trata por tanto de alguien que cumple una obligación no grata y que por tanto será mucho más sensible a las ineficacias y a las pérdidas de tiempo que cuando acude a demandar otro tipo de servicios.

El cumplimiento de una obligación no grata debe ser acompañado con una atención exquisita, personal y material. La Administración Tributaria debe ser capaz de desarrollar con eficacia y simplicidad su tarea, buscando una vinculación con el contribuyente que no agudice la conflictividad de la relación. Debe construirse una organización al servicio de minimizar la "presión indirecta" sobre los contribuyentes, en las que prime el servicio y la colaboración con los cumplidores.

En resumen, podríamos concluir que la Administración Tributaria, cuyo objetivo es la obtención de los recursos necesarios para el cumplimiento de los fines del Estado, a través de la correcta aplicación del ordenamiento jurídico tributario, puede plantearse como misión el logro de la mayor aceptación y colaboración social posible con el Sistema Tributario. 


\section{Misión y líneas estratégicas de la Administración Tributaria}

En el entorno de la gerencia y, más concretamente, de la gerencia pública, la Administración Tributaria se perfila como un espacio lleno de retos y oportunidades. El papel de recaudador que la Administración Tributaria ejercita constituye la faceta fundamental de su relación con los colectivos de contribuyentes. Otras facetas, más amables, quedan parcialmente ocultas por este aspecto. Esta particularidad tensa de la Administración Tributaria convierte a ésta en una organización ciertamente vulnerable ante los medios de comunicación, ante el público en general, e incluso ante otros organismos del sector público y ante sus propios funcionarios, en su faceta de contribuyentes. Este cúmulo de tensiones solamente puede resolverse positivamente incorporando transparencia a las actuaciones, reduciendo los trámites, minimizando las molestias para los contribuyentes cumplidores, con un profundo respeto hacia sus derechos -n definitiva lo que exige es una organización volcada hacia el exterior-, en un entorno de eficiencia en sus actuaciones que transmita una buena imagen, contraria al despilfarro, en la misma administración que recauda -imagen ejemplarizadora de la Administración Tributaria-.

En los últimos años, en consonancia con lo anterior, se está abriendo camino un cambio estratégico en las administraciones tributarias, que conduce a la aceptación del fenómeno tributario por la sociedad como objetivo fundamental de una estrategia que ha sido denominada de ecumplimiento fiscal ${ }^{6}$.

Primera linea. La calidad en el servicio público.-Ofrecer una organización de calidad, centrada en la atención al ciudadanocontribuyente, que facilite el cumplimiento de sus obligaciones fiscales.

Para desarrollar esta línea, la Administración Tributaria debe concentrarse y esforzarse en la materialización práctica de los diversos aspectos, que representan el papel de objetivos operativos respecto al fin último de calidad: a) Gestión volcada hacia el exterior; b) Estatuto y derechos de los contribuyentes; c) Seguridad jurídica; d) Minimizar los costes indirectosn de los contribuyentes. Un conjunto de objetivos que configuran la que hemos denominado Administración Fiscal Receptiva ?

La Administración Tributaria tiene el peligro de generar una cultura introvertida cuyo resultado es una organización impermeable a las necesidades y demandas de los colectivos que se relacionan con ella. En las organizaciones privadas no monopolistas esta situación termina con la disolución de tales organizaciones, cuyo espacio es ocupado por otras que ofrecen servi- cios similares y son más receptivas. La mayoría de las organizaciones del sector público, entre ellas la Administración Tributaria, ostentan una relación monopolística sobre su área de actuación, al tiempo que el contacto del administrado con ellas es insoslayable para éste. Esta situación de asimetría puede provocar una cadena de acciones y reacciones cuyo resultado se concreta en la convergencia de un cierto sentimiento de frustración del administrado, que cultiva una actitud hostil, con la del funcionario que presta el servicio, que reacciona según un mecanismo de mayor distancia hacia el usuario y de rigidez en la aplicación de las normas. Para resolver esta dinámica de distanciamiento es necesario encontrar un elemento de apalancamiento que permita restablecer la fluidez de la comunicación ${ }^{8}$. El elemento de apalancamiento que puede resolver el problema consiste en despertar la receptividad de la organización hacia sus -clientes y usuarios", en este caso hacia los eciudadanos-contribuyentes trasladando la organización hacia el exterior.

Informar al contribuyente de sus derechos y obligaciones en sus relaciones con la Administración Tributaria, contando con un estatuto que enumere los mismos, dentro de los códigos tributarios, constituye un apoyo a la apertura de la Administración Tributaria hacia el exterior y al cumplimiento de sus fines.

"Los contribuyentes tienen derecho a ser informados, asistidos y escuchados; al principio de legalidad en relación con sus obligaciones tributarias; a la seguridad jurídica y a la certeza en relación con sus exacciones fiscales; a recurrir y argumentar; a la tutela judicial efectiva; a la confidencialidad de sus actuaciones...."

La información constituye un deber de la Administración y un derecho inalienable de los contribuyentes, que necesitan saber en todo momento cómo recurrir cualquier decisión administrativa que les afecte. Es más, en una organización tributaria auténticamente abierta hacia el exterior, el contribuyente, además de informado de sus derechos, debería ser asistido por la propia administración en sus reclamaciones, especialmente si el contribuyente en cuestión carece de recursos para acceder al asesoramiento privado. Una Administración Tributaria volcada hacia el exterior no solamente debe informar al contribuyente de sus errores, omisiones o faltas contra la Hacienda Pública, sino que debe informar a éste tan pronto como advierta que el contribuyente ha cometido errores en su propio perjuicio y debe proceder de oficio a la restitución y reparación de los perjuicios que éste haya podido causarse a sí mismo. De esta forma, el efecto inducido por este comportamiento thoneston de la Administración favorece el cumplimiento voluntario de las obligaciones del contribuyente. Así, el principio de seguridad jurídica debe constituir la base sobre las que se asiente la relación contribuyente-Administración.

Dentro de este objetivo de seguridad jurídica" es importante reflexionar sobre el sentido y alcance de diversas fórmulas de 
colaboración con los contribuyentes, a las que nos hemos referido anteriormente.

Un sistema Tributario de masas, que actúa en la complejidad de las presentes relaciones mercantiles y sociales, para desarrollar una gestión y control eficientes, tiene que contar no sólo con la ayuda de las tecnologías de la información sino que debe seleccionar sus espacios de colaboración para optimizar sus recursos. Esto supone una actuación en muy diversos ámbitos, tales como:

- El establecimiento de fórmulas de colaboración amplia para la gestión de los impuestos con diversas entidades (empresas, colectivos de asesores, entidades financieras...).

- La aplicación de sistemas indiciarios y de módulos en actividades económicas de reducida dimensión.

- La existencia de fórmulas, tipo acuerdos previos en áreas muy específicas de potencial litigiosidad y complejidad. En este caso la certeza ofrece valor mutuo tanto para la Administración Tributaria como para el contribuyente.

- El servicio de medios de arbitraje en campos como las valoraciones o las comprobaciones de hechos.

- La existencia de consultas vinculantes en las áreas necesitadas de clarificación por su importancia financiera y el horizonte temporal de las decisiones, fundamentalmente dentro del área empresarial, es igualmente un requisito esencial para la seguridad jurídica en el desarrollo de la economía productiva.

La experiencia de las administraciones más evolucionadas pone en evidencia la correlación existente entre el crecimiento de la recaudación y el desarrollo de los gastos de gestión, que garantizan calidad de atención y percepción, posibilitando la transformación de la imagen tradicional exclusivamente recaudatoria de la Hacienda Pública por la de una administración que desarrolla, asimismo con eficacia, los servicios de información, asesoramiento y resolución de incidencias y litigios que hoy se demandan a la administración financiera ${ }^{10}$.

Finalmente, la complejidad del mundo actual parece consagrar la idea de que las organizaciones funcionan más eficazmente si saben establecer lazos de relación con otras organizaciones que les permitan aprovechar las ventajas comparativas, que poseen debido a su campo de especialización. En este sentido, la Administración Tributaria, en línea con lo ya expresado con anterioridad, debe aprender a beneficiarse de la aportación de muy diversas entidades colaboradoras en la gestión (empresas, entidades financieras) y también en la aplicación (gestores, asesores fiscales, corporaciones económicas y sociales) para facilitar el cumplimiento fiscal; todo ello con las debidas garantías para el interés público y los mecanismos de control correspondientes.
Pero la calidad en la gestión no puede limitarse a la atención de los contribuyentes. La calidad en la gestión debe transcender al conjunto de las actuaciones de la Administración Tributaria. La adecuación de medios escasos a fines obliga a la puesta en práctica de la administración por objetivos y al desarrollo específico de indicadores de eficiencia. La aplicación de métodos de evaluación conjunta de las tareas, que como política pública ejerce, pueden ser utilizadas con éxito en nuestras organizaciones. La política de gestión y control de grandes contribuyentes, la articulación territorial de esa política, los programas de control en oficina, los planes de inspección, el tratamiento de los medianos contribuyentes, las áreas de riesgos "fiscal y un largo etc... de actuaciones necesitan de una planificación estratégica y de una programación y control de objetivos. Todo ello ligado a las políticas de integración y recursos humanos. La calidad en este sentido como suma de cumplimiento de objetivos y eficiencia es esencial, como en cualquier otra organización, al quehacer global diario de la Administración Tributaria.

Segunda linea. Lucha contra el fraude fiscal.-La estrategia de cumplimiento fiscal pasa, asimismo, por una lucha efectiva contra el fraude fiscal. Parte del esfuerzo para desarrollar una relación más correcta y satisfactoria entre la Administración y los ciudadanos que cumplen sus obligaciones tributarias consiste en "asegurar y garantizar que todos los contribuyentes son (y serán cada vez más) impulsados a cumplir con sus obligaciones" $"$.

La propuesta de cumplimiento fiscal "voluntario" no significa un debilitamiento de la fiscalización o inspección fiscal, ni tampoco de las potestades sancionadoras. Muy al contrario, debe significar la persecución sistemática del fraude y, en consecuencia, el desarrollo de una atención especial en la gestión y en la investigación destinados a su dificultación y hacia la neutralización de los mecanismos que lo hacen posible.

Tradicionalmente, las Administraciones Tributarias han tendido a identificar gestión tributaria con cumplimiento "voluntarion, y fiscalización o inspección con lucha contra el fraude. Pero esto no es del todo cierto. En las Administraciones Tributarias modernas está plenamente demostrado que la gestión es básica en la lucha contra el fraude. Dentro de los modelos tributarios de "masas", a través de los controles cruzados de información, se pueden lograr objetivos muy amplios en contra de la defraudación. Los procesos de gestión tienden a aespecializarse en los "pequeños fraudes más generalizados, mientras que la fiscalización debe tender a pivotar sobre las áreas de riesgo más específicas y complejas.

Para vincular la actividad inspectora y de gestión a la estrategia de cumplimiento fiscal, se ha acuñado un concepto operativo: Presencia Inspectora. aLa presencia inspectora descansa sobre la percepción social del poder de la inspección, que con- 
siste en la sensación de riesgo de ser descubierto y castigado en caso de defraudar fiscalmente.

Dicho concepto implica en sus consecuencias a todos los ciudadanos-contribuyentes, englobando a las categorías de ciudadanos potencialmente cumplidores e incumplidores. A estos últimos se les transmite la sensación de riesgo y, a los primeros (parcialmente coincidentes), una señal inequívoca respecto al riesgo y un mensaje positivo en cuanto a la defensa de sus intereses.

Esta concepción parte de cierta evidencia empírica conectada con la teoría denominada de las -Expectativas Racionales` y de la :Utilidad, que incluye la aversión al riesgo como factor fundamental de configuración del comportamiento humano. El cumplimiento fiscal no se debería únicamente a consideraciones morales o sociales condenatorias respecto a la evasión, ya que el comportamiento de los contribuyentes varía, en los experimentos realizados, ante instrucciones eneutrales" evaluadoras de riesgos, sin especificar, e instrucciones antencionadas de riesgos ${ }^{12}$.

Los efectos del impacto de la presencia inspectora exigen una distinción entre efectos de naturaleza directa y efectos de naturaleza indirecta.

Se puede cuantificar el efecto directo de la presencia inspectora mediante un índice de seguimiento de contribuyentes seleccionados que incorpore una medida de la deuda descubierta, algún ratio expresivo de la calidad de la fiscalización y el número de personas inspeccionadas e investigadas.

La cuantificación del efecto indirecto exige que se tenga en cuenta el cumplimiento "voluntario" inducido para el conjunto de los contribuyentes, incorporando la consideración de la percepción social del poder de la Administración Tributaria. Son escasos los estudios empíricos con los que contamos actualmente, en relación con los efectos inducidos de la presencia inspectora. Posiblemente, uno de los más completos se refiere al efecto de las actuaciones inspectoras sobre la recaudación en el Impuesto Federal sobre la Renta en Estados Unidos durante el período 1977-1986 ${ }^{13}$.

En Estados Unidos, la década 1977-1986 fue testigo de un impresionante decremento en el porcentaje de inspecciones realizadas por la Inspección de los Tributos. El porcentaje de inspecciones sobre declaraciones personales varió desde el $2,5 \%$ hasta poco más del $1 \%$. El porcentaje de inspecciones sobre declaraciones de sociedades decreció desde el 9,5\% hasta el $3 \%$. El estudio citado corrobora el papel central desempeñado por el porcentaje de actuaciones inspectoras sobre el nivel de recaudación fiscal. Los recursos adicionales que se invierten en investigación e inspección reflejan una productividad marginal muy elevada. Un hecho que podría haber quedado oculto hasta ese momento, debido a la aparente estabilidad del sistema recaudatorio a lo largo del tiempo.
La realización de planes específicos de lucha contra el fraude, que incorporen acciones pluri-anuales y operen en campos tan diversos como la percepción social, la comunicación, la educación, la coordinación administrativa y gestora, las reformas legislativas, los planes integrados de gestión e inspección... pueden resultar muy útiles y permiten alcanzar buenos resultados también en el corto plazo.

Otros instrumentos operativos, como las regularizaciones fiscales, tienen la virtud de consolidar espacios de •normalización tributaria., pero no están exentos de peligro si no se programan minuciosamente y se realizan muy excepcionalmente. Los peligros se encuentran ampliamente documentados y consisten fundamentalmente en el riesgo de que las expectativas de afloramiento de dinero oculto no se cumplan, con el consiguiente desprestigio para la Administración Tributaria que promovió el programa; que se generalice entre segmentos importantes de contribuyentes la percepción de un menor cumplimiento futuro por parte de aquellos que venían respetando sus obligaciones fiscales, y que se generalice la percepción social de incumplimiento a través de procesos continuados de ofertas de normalización que deterioran la utilización de este instrumento. No obstante, la estrategia de aregularización fiscaln, articulada con un plan específico de comunicación externa dirigido selectiva y diferencialmente a los distintos segmentos de contribuyentes, junto a una presencia inspectora coordinada, puede resultar, en determinados contextos históricos o políticos, extraordinariamente positiva, dentro de la estrategia de normalización y cumplimiento fiscal voluntario.

En consecuencia, puede ser un paso necesario, según se desprende de los incipientes desarrollos de la aTeoría de las Amnistías Fiscalesn y las experiencias de los proyectos de regularización fiscal (por contraposición a amnistía) desarrollados en distintos países, con éxito variable ${ }^{14}$.

El nuevo objetivo de la Administración Tributaria consiste en promover y fomentar una alianza implícita entre la Administración y los ciudadanos que cumplen con las normas fiscales, en contra de aquellos que defraudan al Estado y a la sociedad. Este sistema de alianzas representa un elemento básico en una estrategia dirigida a fomentar el cumplimiento voluntario de las leyes tributarias, y debe configurar asimismo la acción de auditoría y verificación fiscal y las estrategias de uregularización tributaria".

Finalmente es necesario, en este epígrafe dedicado a la lucha contra el fraude fiscal, referirse específicamente a la problemática que en este campo incorpora los crecientes procesos de descentralización territorial de competencias, de integración económica supranacional y de internacionalización de la economía. Las posibilidades para la evasión y el fraude fiscal que esta realidad incorpora obliga a las Administraciones Tributarias a dedicar una especial atención a los procesos de información, 
control y colaboración con otras Administraciones nacionales. Tanto el diseño de sistemas de información y gestión compartidos (descentralización territorial y procesos de integración supranacional), como los procesos de consulta e información a propuesta de parte $y$, de forma muy destacada, la verificación inspectora (fiscalización) compartida (empresas supranacionales, contribuyentes con puntos de conexión territorial diversa). Todos estos procesos necesitan de una cultura y una atención organizativa muy precisas en cada país, para su desarrollo eficiente.

Tercera Línea. El impulso de un compromiso institucional y social.-Ya nos hemos referido anteriormente a que el elemento básico que caracteriza la actuación de la Administración Tributaria es la detracción de recursos de las economías privadas para ponerlos a disposición de los poderes públicos. Por tanto, la Administración Tributaria ejerce su actividad en un campo esencialmente conflictivo tanto en relación con el contribuyente individual como en el contexto de las relaciones sociales. En primer lugar, la acción tributaria se dirige como hemos visto al conjunto de la sociedad, por lo que el grado de colaboración y aceptación social del Sistema Tributario es un fenómeno claramente interdependiente, en el que muy diversos agentes económicos y sociales actúan e influyen en la percepción que del fenómeno tributario tiene el conjunto de la sociedad ${ }^{15}$. La capacidad de actuación unilateral de la Administración Tributaria es muy limitada, por lo que hemos señalado que debe combinar la acción propia, a través de la comunicación externa hacia la sociedad, con la colaboración e incidencia en aquellos sectores sociales que de forma más precisa influyen en la aceptación general del modelo tributario. Junto a los factores relacionados con las expectativas racionales y utilidad individualn, es preciso señalar los factores sociales, ciudadanos, éticos, corporativos, legislativos, políticos y judiciales, que se encuentran también intimamente vinculados al cumplimiento fiscal, por los ciudadanos, de las obligaciones tributarias. En consecuencia y como expresión de algunos de los campos en que puede materializarse e incidir la acción estratégica de la Administración Tributaria podemos destacar: 1) En el campo legislativo, informando y asesorando a los legisladores para conseguir un ordenamiento jurídico-tributario que sea aplicable socialmente, con normas tributarias integradas y simples que posibiliten un alto grado de "eficiencia fiscaln, que minimicen la posibilidad del fraude, y técnicamente coherentes. 2) En el campo del conjunto de la Administración Pública, ligando el pago de impuestos a la provisión de los servicios adecuados y a la satisfacción de las necesidades sociales, ya que resulta notoria la mejora en el cumplimiento fiscal si se ofrece una percepción social de eficacia en el gasto público, lo que implica una acción tendente a que las unidades de gasto se preocupen por esta imagen de eficacia y que la percepción de los ciudadanos acerca del uso de los re- cursos aparezca como adecuado. 3) En el campo judicial, debe atenderse a la difusión de las normas tributarias entre los organismos judiciales y propiciar una colaboración constructiva que reduzca el plazo de las sentencias judiciales, favoreciendo su ejemplaridad. 4) El campo corporativo es un conjunto institucional especialmente importante, ya que permite la colaboración con sindicatos y organizaciones empresariales, para que la ciudadanía acepte el fenómeno tributario como un hecho saludable e inherente a la organización democrática de la sociedad. Asimismo, la regulación de la figura del asesor fiscal, definiendo su papel y responsabilidades ante la sociedad y ante la Administración, resulta de significativa importancia, ya que es un colectivo de intermediación entre la Administración Tributaria y el contribuyente. De igual forma, la colaboración con los diversos colectivos profesionales de abogados, economistas, censores, etc., representa una vía instrumental de afianzamiento y sintonía social con el fenómeno tributario. 5) Estas líneas de colaboración tienen una extensión básica en la educación escolar y en la interconexión con los centros de formación universitarios, la formación profesional y el mundo doctrinal, en los que es imprescindible una presencia permanente que favorezca la normalización en la aplicación cotidiana de las normas tributarias y una cobertura positiva en relación a las nuevas reformas. 6) Los medios de comunicación constituyen un instrumento de transmisión y amplificación que, por la especial sensibilidad pública ante el hecho de la imposición, impone que las relaciones de la Administración Tributaria con los mismos estén presididas por la fluidez y la ausencia de sensacionalismos perjudiciales para la imagen del hecho fiscal. Y 7) En una esfera más social y ciudadana, la inadecuación en lo que podríamos denominar "moral social", solidaridad y valores de ética tributaria, a través de la búsqueda de la colaboración activa de las instituciones morales y ciudadanas más relevantes.

En todos estos campos la Administración Tributaria debe procurar incidir socialmente. En consecuencia, la Administración Tributaria no sólo puede concebirse como una organización técnica de gestión de sistemas de información y aplicación económica y jurídica de hechos imponibles, sino también como una organización social, que forma parte de lo "públicon y del Estado de Derecho en primera línea de fuego. En un entorno en que el poder coercitivo que ejerce es fundamental, pero en el que de igual manera también lo son los factores de legitimidad de ejercicio y consenso social vinculados a los modelos de democracia participativa y de poderes sociales compartidos en los que se desenvuelven las sociedades contemporáneas.

Cuarta linea. Construcción de una organización integrada.La vertebración de una organización caracterizada por sus atributos de coerción y generalidad, su dimensión social y su carácter de instrumento financiero, con que la sociedad se dota a sí misma para cumplir con el objetivo de legitimidad de la ac- 
ción política, exige que sus empleados tengan el concepto de misión de la organización tributaria y compartan la visión y valores éticos y de responsabilidad pública, orientación al servicio, orientación hacia el exterior, asistencia al ciudadano-contribuyente, lucha contra el fraude, calidad en la prestación del servicio..., ya que al ser una relación de carácter no voluntario la buena calidad en el servicio redundará en la mayor aceptación del hecho tributario y de la administración encargada de su gestión. Para que esta identificación cultural de los miembros de la organización sea posible, dos instrumentos resultan imprescindibles: uno es la formación y educación de los empleados de la Administración Tributaria en estos valores, en la comprensión de la misión organizativa y en la difusión de una pauta cultural homogénea que impulse el sentido de pertenencia a la organización; el otro elemento consiste en un sistema de incentivos, y un modelo organizativo y técnico, que mantenga la tensión y el interés de los funcionarios y empleados, y canalice los esfuerzos de los mismos en favor de la organización ${ }^{16}$

\section{Cinco conceptos de gestión de organizaciones aplicables a la Administración Tributaria}

\section{Los conceptos de organización estratégica y de complejidad}

Si hay una organización estratégica por excelencia ésta es la Administración Financiera del Estado. Tanto la Administración Tributaria, como la Presupuestaria en sentido amplio, son organizaciones vinculadas al funcionamiento estructural de la sociedad. Sus decisiones resultan extraordinariamente relevantes para el quehacer económico-social, político e institucional. Como toda organización estratégica combinan importantes compromisos en el corto plazo con escenarios de oportunidad en un horizonte temporal más amplio. Operan en un entorno de complejidad y diversidad extraordinario e integran un numeroso plantel de profesionales y gestores que realizan una multitud de tareas "productivas", "de información", "servicio", "estudion, "controln...

Forman parte del núcleo "duro" del Estado, actuando en escenarios de conflicto ideológico, social y administrativo notorio.

Tienen que tomar decisiones que les permita afirmar misiones tales como el "cumplimiento fiscal voluntarion, para lo cual tienen que establecer sólidos planes de lucha contra el fraude fiscal y modelos de cumplimiento y calidad para el conjunto de los contribuyentes. O en otros casos combinar modelos de presupuestos y control, rígidamente estabilizadores para las finanzas públicas, con instrumentos favorables a la flexibilidad y a la gestión eficiente de políticas públicas.

En fin, tienen que aprender a ser eficaces en la gestión cotidiana, dura, difícil, conflictiva, construyendo a la vez Instituciones eficientes adaptadas a la evolución de la Sociedad, sobre la que influyen con su propia dinámica de cambio.

El carácter estratégico de la organización conlleva a menudo la necesidad de aplicar el pensamiento estratégico a muchas de sus decisiones: las políticas públicas y su control, la tributación de las actividades empresariales y comerciales modestas, los programas de lucha contra el fraude, son algunas de las acciones que implican aplicación de modelos y decisiones de agestión estratégican. La Administración Financiera desarrolla dos de las más importantes políticas públicas de un Estado de Derecho: la regulación y administración del Sistema Tributario y la política presupuestaria.

\section{El concepto de organizaciones "aprendientes"}

Mucho se ha escrito sobre el papel del conocimiento en las sociedades contemporáneas. La capacidad adaptativa es por otra parte una prioridad para alcanzar los objetivos organizativos en un mundo en que el cambio y lo imprevisto se encuentra de forma preeminente en las agendas de las organizaciones. El concepto de organización "aprendiente" sintetiza esta doble necesidad. De un lado, crea las condiciones para que el conocimiento fluya entre las diversas unidades y personas y se transforme en conocimiento compartido de la organización. De otro, los mecanismos de una dirección y gestión que contemplan una cultura de participación, y comparte la reflexión y la acción con los cuadros intermedios de la organización, permiten destilar los conocimientos y favorecer la inclusión en los planes organizativos de un conjunto activo y amplio de recursos humanos, identificados con la organización y por tanto capaces de afrontar las iniciativas necesarias para adaptar la organización a la complejidad imprevisible de lo cotidiano y a los escenarios de mejora en la calidad y el cumplimiento de objetivos.

La formación aparece, en consecuencia, como un factor que transciende del aaprendizaje técnicon para inscribirse en el núcleo central de la dirección y cultura organizativa. Esto implica, al menos, dos consecuencias: de una parte, la formación se transforma en factor estratégico dentro de la política de recursos humanos y, de otra, supera las barreras de los aprocesos reglados" para incrustarse en el mecanismo de dirección y toma de decisiones de la organización. Las organizaciones devienen en "aprendientes", la formación en factor "orgánico". La formación en su sentido tradicional descubre de esta forma sus pro- 
pios -límites". Aprender a dirigir dirigiendo; hacer de la gestión una forma de aprendizaje colectivo; son algunos de los amitos" ligados a este paradigma de organizaciones aprendientes", que está transformando notablemente el trabajo en las organizaciones en este fin de siglo.

\section{La gestión como proceso de negociación}

Ningún gestor público y desde luego ningún gestor de la Administración Tributaria, cuenta por sí mismo con los instrumentos necesarios para el logro de sus objetivos. Los mismos dependen igualmente de una multitud de sujetos personales e institucionales, internos y externos a la Administración Tributaria, que a menudo influyen decisivamente en el cumplimiento o alejamiento de nuestros fines. Nuestros colaboradores, nuestros jefes, nuestros colegas de otras dependencias y departamentos, las asociaciones de contribuyentes, los asesores fiscales, los jueces, los medios de comunicación, y un largo etc., condicionan el éxito o fracaso de nuestro trabajo. En un mundo interdependiente, en que "todo depende de todo" y en el que a menudo compartimos responsabilidades y competencias, la capacidad para "compartir objetivos" aparece como una buena fórmula de éxito organizativo. De igual manera, una buena agestión de los conflictos" aparece como imprescindible. Crear objetivos compartidos y gestionar el conflicto se encuentra plenamente vinculado a los procesos de negociación, y en consecuencia a la capacidad para crear y reclamar valor con el resto de sujetos actuantes. La construcción de espacios de amutuo interésn y fórmulas de coordinación y "complicidad", las iniciativas para establecer acuerdos duraderos, aparecen como imprescindibles.

\section{La dirección como integración}

Crear perspectivas a nuestras organizaciones, ilusionar con proyectos de futuro, "saber a donde se va", pero a la vez remo- ver los obstáculos que dificultan la gestión cotidiana, entusiasmar con lo inmediato, formar en una cultura de calidad y eficiencia. Estos serían algunos de los principales objetivos de cualquier directivo. Pero sobre todo la gestión es integración de tareas y personas, formación de equipos, perspectivas unitarias para la organización. La Administración Tributaria es una organización compleja de un marcado carácter estratégico, necesita su visión y objetivos unitarios, la recaudación, la gestión en sentido estricto, la inspección, las aduanas no son unidades separadas con lógicas independientes. Los objetivos unitarios, los planes estratégicos son esenciales para que a nivel sectorial y territorial exista una gestión vertebrada. Pero más allá de estos aspectos organizativos la integración supone política compartida de recursos humanos, de comunicación, de valores, de identificación con la organización global. El directivo en cualquier nivel es un elemento orgánico integrador de conocimientos, habilidades, personalidades, aportaciones, sectores, al tiempo que, funcionalmente, es el organo decisor. Combinar integración y decisión de forma inteligente es sin duda el gran reto.

\section{El papel de la ética y de los valores}

En la actualidad, existe una preocupación creciente en las organizaciones por los valores y la ética integrados en la cultura corporativa. La Administración Financiera como parte de la Administración Pública está obligada a dedicar una atención especial a la ética y a los valores de responsabilidad pública. No nos referimos aquí a normas legales imprescindibles ni a medidas anticorrupción, incompatibilidades y otros conceptos análogos de gran importancia. La necesidad de construir de forma compartida unos valores de responsabilidad pública ligados a una cultura institucional, profesional y de respeto a las personas, es un complemento de las normas y la única garantía para su interiorización por la organización. Dedicar una especial atención a la formación participativa en estos valores es imprescindible en una organización pública que opera en un núcleo tan sensible para la legitimidad del Estado y sus tareas.

\footnotetext{
- Este trabajo forma parte del primer capítulo del Libro Lecturas de Gerencia desde la Administración Financiera; de próxima publicación por el Instituto de Estudios Fiscales.

- Director del Instituto de Estudios Fiscales

1 Curtis Ventriss, Hacia una filosofia püblica de la Administración Puiblica: una perspectiva civica de lo público. Universidad de Vermont (California)

2 Larry D. TeRrY, Revista de Administración y Sociedad, liderazgo en la Administración.
}

\footnotetext{
3 Michael Barzelay y José María O’Kean, .Gestión pública estratégica, conceptos, análisis y experiencias: el caso IPIA. I.E.F. Monografia 71. Madrid, 1989.

4 Ver la obra de Eric MONNIER, Evaluación de la acción de los poderes públicos. Colección de Estudios de la Hacienda Pública. Edición en espanol Instituto de Estudios Fiscales 1995.

' O.C.D.E., Études sur la gestion publique. París, 1988.

6 Para un seguimiento de este cambio estructural, véanse las ponencias de la Conferencia Técnica del CIAT de Munich: Responsabilidad gerencial del administrador
} 
Iributario. Consültense, también, las ponencias correspondientes a la XXV Asamblea General del CIAT: Estrategias de la Administración Tributaria para la década de los 90. Centro Interamericano de Administradores Tributarios. Instituto de Estudios Fiscales. Madrid 1989-1992.

- El primer paso hacia la receptividad consiste en acrecentar la calidad de la acogida y la celeridad de los servicios en los puntos de contacto con el público, así como en el perfeccionamiento y mejora de la comunicación con los contribuyentes, que deberán comprender el porqué de los impuestos y percibir a las agencias fiscales como servicios que aseguran a cada uno su justa contribución. En segundo lugar, existen actitudes y comportamientos de quienes atienden las ventanillas, OCDE. Comité de Cooperación Técnica y Comité de Asuntos Fiscales. La receptividad administrativa y el contribuyente. Un resumen para los decisores. Paris, 1989.

8 Peter M. Senge, La Quinta Disciplina. Ed. Granica. Barcelona. 1992.

9 Para un mejor conocimiento de la práctica en los distintos paises, en relación con esta problemática, consúltese el documento del Comité de Asuntos Fiscales de la OCDE, Los Poderes de la Administración Fiscal y las Derechos del Contribuyente. París, marzo 1990. En este documento se pasa revista a los aspectos aludidos y a otros como la figura del defensor del contribuyente y los programas de educación e información en las escuelas.

10. Para el análisis del objetivo de calidad dentro de la estrategia de mejora del cumplimiento fiscal y del proceso de cambio en las administraciones tributarias, para su consecución, véase el documento Una estrategia para la modernización de la Administración Tributaria. Instituto de Estudios Fiscales. Madrid, mayo 1991.

"Una estrategia para ... Ob. cit. Instituto de Estudios Fiscales.

12 Un estudio empírico de esta naturaleza ha sido realizado en la Universidad de
Colorado (USA) por James ALm, Gary H. McCleunsd y Willam D. Sohuzze, publicado bajo el título Why People pay Taxes?

13 Véase Jeffrey A. Dubls, Michael J. Graetz y Louis L. Wibe. The effect of Audit Rates on the Federal Individual Income Tax 1977-1986. National Tax Journal. Vol 63, $n^{\circ}$ 4, dicbre. 1990. En este trabajo se analizan los efectos del porcentaje de inspecciones realizadas por el IRS, junto a otros factores, sobre el porcentaje de declaraciones individuales presentadas en el periodo voluntario, asi como sobre el número de declaraciones complementarias. El estudio concluye que las cuotas declaradas en 1986 se hubieran incrementado en quince mil millones de dólares si el número de inspecciones realizadas se hubiera mantenido a los niveles de 1977 durante el periodo considerado, que coincidió con un momento de fuertes restricciones presupuestarias.

is Véase en esta misma obra, Teodoro Cordón EzQuerro, -Dos Ejemplos sobre la Aplicación del Pensamiento Estratégico en la A.E.A.T.

is Véase Fernando Díaz YuBERo y Juan A. Garde RocA, Nuevas concepciones sobre el contenido de la gestión pública y la Administración Tributaria.

Véase, también, Ignacio Ruiz-Jarabo, Gestión de la Hacienda Pública y comportamiento social. Curso de Gerencia Pública. Escuela de la Hacienda Pública. Madrid, 1989.

${ }^{16}$ No es éste el lugar adecuado para efectuar un análisis de la rica experiencia organizativa y de gestión de las administraciones tributarias. El conjunto de materiales editados por el Instituto de Estudios Fiscales, correspondientes a las conferencias técnicas y asambleas anuales del Centro Iberoamericano de Administración Tributaria (C.I.A.T.) permiten un trabajo de investigación de enorme vigencia. El funcionamiento de las administraciones tributarias puede estudiarse asimismo en la obra colectiva La Administración Tributaria en los Paises del CIAT. Instituto de Estudios Fiscales. Madrid, 1992. 


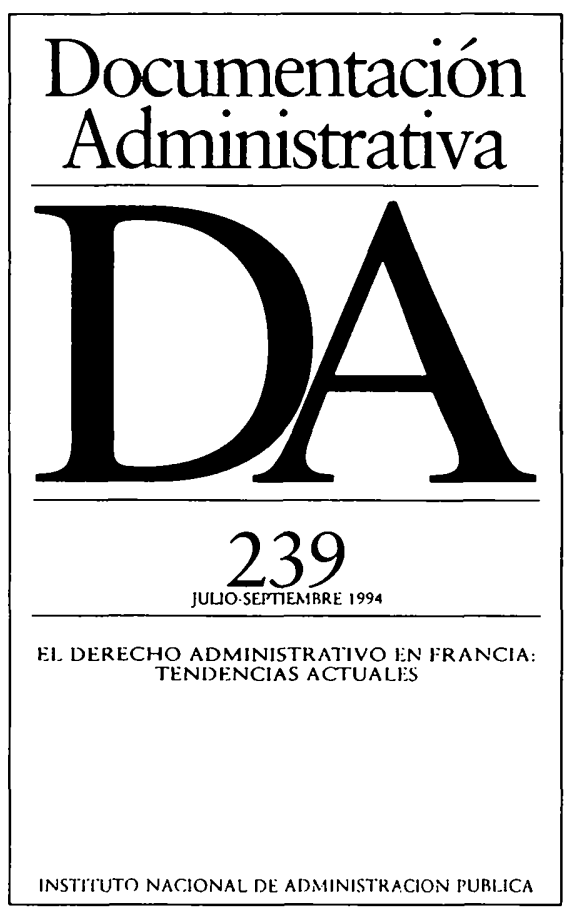

Publicación trimestral del Instituto Nacional de Administración Pública, especializada en Derecho Administrativo y Organización y Gestión de las Administraciones Públicas.

Aparecida en 1958, la característica más destacada de su actual etapa es la dedicación monográfica de cada número a una cuestión específica, que se analiza desde diferentes puntos de vista por acreditadas firmas de España y otros países.

\section{CONSEJO DE REDACCIÓN}

Presidente: Alejandro Nieto García. Vocales: Manuel Aragón Reyes, Enrique Argullol Murgadas, Miguel Beltrán Villalva, Andrés de Blas Guerrero, José Manuel Castells Arteche, Rafael Gómez-Ferrer Morant, Luis Morell Ocaña, José Ramón Parada Vázquez, Juan Alfonso Santamaría Pastor, Gumersindo Trujillo Fernández.

\section{Director}

Luciano Parejo Alfonso

\section{Secretario}

Jesús Prieto de Pedro

\section{Edita:}

Instituto Nacional de Administración Pública ISSN: 0012-4494

NIPO: 329-94-001-5

Depósito legal: M 581-1958

\section{Distribución y suscripciones:}

Instituto Nacional de Administración Pública Subdirección General de Documentación, Investigación y Publicaciones Atocha, 106. 28012 MADRID

Teléfono: (91) 3493104 Fax: (91) 3493287

\section{Precios:}

Suscripción anual ( 4 números) .... 4.000 pts. + IVA
Número sencillo ............................. 1.300 pts. + IVA
Número doble ............................ 2.600 pts. + IVA
Más 300 pts. de gastos de envío

